\title{
Patrones espaciales en comunidades de macroinvertebrados acuáticos de la Puna Argentina
}

\author{
Carolina Nieto ${ }^{1,3}$, Agustina Malizia ${ }^{2,3}$, Julieta Carilla ${ }^{2,3}$, Andrea Izquierdo ${ }^{2,3}$, José Rodríguez ${ }^{1}$, \\ Soledad Cuello ${ }^{3,4}$, Martín Zannier ${ }^{2} \&$ H. Ricardo Grau ${ }^{2,3}$ \\ 1. IBN- Instituto de Biodiversidad Neotropical. Facultad de Ciencias Naturales e IML, Universidad Nacional de \\ Tucumán. Miguel Lillo 251. Tucumán. Argentina. UNASUR (CP 4000); macarolina_nieto@yahoo.com.ar, \\ josephum@hotmail.com \\ 2. IER- Instituto de Ecología Regional, Facultad de Ciencias Naturales e IML, Universidad Nacional de Tucumán. \\ Casilla de correo 34, Yerba Buena (CP 4107), Tucumán, Argentina; agustinamalizia@yahoo.com, \\ julietacarilla@gmail.com, aeizquierdo@gmail.com,martinzannier@hotmail.com,chilograu@gmail.com \\ 3. CONICET. \\ 4. Instituto de Química del Noroeste Argentino (INQUINOA). CONICET. Universidad Nacional de Tucumán; \\ asolecue@gmail.com
}

\section{Recibido 16-IV-2015. Corregido 10-XII-2015. Aceptado 13-I-2016.}

\begin{abstract}
Spatial patterns in communities of aquatic macroinvertebrates of Argentinean Puna. The macroinvertebrates are a vital component of freshwater ecosystems as they contribute to the process of organic matter while serving as food for other organisms such as fish and amphibians. Unfortunately, the knowledge of the aquatic diversity is poor in the high Andean systems (between 3200 and 5200 m.a.s.l. and rainfall below $300 \mathrm{~mm}$ per year), especially in the Argentinean peatbogs, a fact which has made difficult the interpretation of spatial patterns on a regional scale. The present study aimed to describe the composition of aquatic macroinvertebrates in seven peatbogs in the Argentinean Puna, and to analyze for the first time their spatial patterns. For this, we studied the relationship of these organisms with the environment, and obtained information about the surrounding vegetation and water physico-chemical characteristics. A total of 3131 specimens of aquatic macroinvertebrates were collected, representing 25 taxa belonging to 22 families and 24 genera. In addition, 62 species of vascular plants were recorded, belonging to 20 families. The most abundant life form were the tufted grasses, followed by cushions. By using an NMDS (Non-Metrical Multidimensional Scaling) statistical analysis, the sampling sites were ordered in each peatbog as follows. The peatbogs located furthest West and South with higher water temperature were grouped on axis 1, whereas those with higher conductivity, whole water dissolved solids and salt concentration were grouped on axis 2 . The water temperature was higher than air temperature at all times, and we found no association between temperature and altitude. The altitude had no correlation with the abundance of macroinvertebrates or with plant richness. Life forms such as scattered graminoids, trailing and prostrate herbs (in many cases they get into the channel) and aquatic plants were more abundant in peatbogs 4 , 5 and 7 and they had a correlation with some macroinvertebrates belonging to functional trophic groups such as filter-collectors, collectors and scrapers. Finally, conductivity, whole dissolved solids and salt concentration had their highest value in peatbog 6, where Bivalvia (filter-collector) and Hyalella (collector) were also found. The results obtained attest that these macroinvertebrates displayed diversity and composition spatial patterns, the most important feature being their relationship with the surrounding vegetation, and to a lesser extent, with the physical and chemical traits of water in seven fertile lowlands in the Argentinean Puna. Rev. Biol. Trop. 64 (2): 747-762. Epub 2016 June 01.
\end{abstract}

Key words: macroinvertebrates, peatbogs, vegetation, aquatic ecosystems, puna, neotropical. 
Los macroinvertebrados son un componente vital en los ecosistemas acuáticos. Los estados inmaduros y en muchos casos también los adultos, forman parte de diferentes grupos tróficos, tales como colectores-recolectores, colectores-filtradores, trituradores, raspadores, e incluso predadores (Domínguez, Molineri, Pescador, Hubbard, \& Nieto, 2006; Reynaga \& Dos Santos, 2012). De este modo, los macroinvertebrados acuáticos juegan un rol fundamental en el reciclado de nutrientes, procesando enormes cantidades de materia orgánica y sirviendo también de alimento para otros organismos como los peces o anfibios (Giorgi \& Tiraboschi, 1999). Además, gran parte de la transferencia de biomasa hacia los ecosistemas terrestres es realizada por las formas adultas de los insectos acuáticos, después de abandonar el agua, al actuar como presas, como depredadores o sólo por reproducirse, subsidiando energéticamente las cadenas alimenticias terrestres (Wesner, 2012). Las eclosiones masivas de insectos transfieren grandes cantidades de fosfato y nitrato hacia los ecosistemas terrestres ayudando a la eliminación y limpieza de contaminantes de los sistemas acuáticos (Edmunds, Jensen, \& Berner, 1976; Domínguez et al., 2006).

Los sistemas acuáticos andinos incluyen una amplia variedad de ambientes, desde pequeños a grandes lagos de distintos orígenes, nacientes de ríos que en muchos casos se asocian a grandes humedales o vegas, $y$ ambientes extremos tales como lagos salinos y manantiales termales (Maldonado et al., 2011). Estos sistemas hídricos andinos tienen en común ciertas características como ser: temperaturas medias anuales bajas (alrededor de $10^{\circ} \mathrm{C}$, Wasson, Guyot, Dejoux, \& Roche, 1989); una fuerte amplitud térmica diaria de hasta $20^{\circ} \mathrm{C}$ (Wasson et al., 1989); baja presión parcial atmosférica que indicaría también una baja disponibilidad de oxígeno disuelto en el agua; altitudes elevadas con una topografía muy compleja y cobertura vegetal de ribera normalmente escasa, representada por formaciones de herbáceas de páramo, puna y relictos de bosques (Jacobsen, 2008a). La semejanza entre estos sistemas hídricos andinos y los ecosistemas fluviales templados alpinos ha sugerido que las comunidades de macroinvertebrados podrían ser más semejantes a sus contrapartes templadas alpinas, que a los sectores más bajos de los mismos ríos tropicales o subtropicales (Jacobsen, Schultz, \& Encalada, 1997).

En los sistemas altoandinos, la diversidad acuática ha recibido mucha menos atención que la diversidad terrestre, lo que ha dificultado la interpretación de patrones espaciales a escalas regionales (Maldonado et al., 2011). En general, son pocos los trabajos realizados para esta región, por ejemplo Rumrich, Lange-Bertalot, \& Rumrich (2000) trabajaron con diatomeas andinas, Donato (2001) con algas de Colombia, y en los casos de Maldonado-Ocampo et al. (2005) en Colombia, Ortega \& Hidalgo (2008) en Perú y Sarmiento \& Barrera (1997) en Bolivia trabajaron con peces. Con respecto a los macroinvertebrados altoandinos, Jacobsen (2008a) y Maldonado et al. (2011), realizaron una síntesis de lo que se conoce a nivel de comunidades de altura de Bolivia, Colombia, Ecuador y Perú. Concluyeron que el patrón de diversidad decreciente cuando se incrementa la altitud, es menos claro en los macroinvertebrados respecto a otros grupos como los peces (Jacobsen, 2008a), y que dentro de la comunidad de los macroinvertebrados, los insectos son usualmente los dominantes tanto en su taxonomía como en su cantidad (Jacobsen, 2008a).

En Argentina son escasos los estudios de macroinvertebrados que habitan sobre los 2000 $\mathrm{m}$ de altura; la mayoría están basados en un grupo taxonómico en particular. Por ejemplo, Scheibler, Pozo, \& Paggi (2008) analizaron la distribución espacio-temporal de Chironomidae en un arroyo andino de Mendoza a lo largo de $700 \mathrm{~m}$ (de 1700 a $2200 \mathrm{msnm}$ ), y Tejerina \& Malizia (2012) evaluaron la comunidad de Chironomidae en una cuenca subtropical de Tucumán a lo largo de un gradiente altitudinal de $1500 \mathrm{~m}$ (de 700 a $2100 \mathrm{msnm}$ ). En el caso específico de las vegas o bofedales de Argentina (entre los 3200 y $5200 \mathrm{msnm}$ y precipitaciones menores a los $300 \mathrm{~mm}$ anuales) el 
conocimiento de las comunidades de macroinvertebrados es escaso o nulo.

Las vegas se localizan sobre topografías cóncavas, con alta humedad edáfica combinada con bajas tasas de descomposición asociadas a bajas temperaturas, lo que produce un tipo particular de vegetación y suelos. Aunque representan aproximadamente sólo el $0.66 \%$ de la superficie total de Argentina, las vegas de alta montaña constituyen unidades funcionales claves en las regiones puneñas y altoandinas (Izquierdo, Foguet, \& Grau, 2015), dado que concentran el mayor porcentaje de la biodiversidad de estas ecorregiones y de ellas depende fuertemente la regulación de los ciclos hidrológicos, los flujos de carbono, y la provisión de recursos para los grandes herbívoros nativos y exóticos (Halloy, 1991; Seimon et al., 2007); por lo que son consideradas unidades prioritarias de conservación.

En este trabajo, se realizó la primera descripción cuantitativa de los patrones espaciales de diversidad y composición de macroinvertebrados acuáticos en siete vegas de la Puna argentina, y su relación con las características del ambiente, de la vegetación circundante y con características físico-químicas del agua.

\section{MATERIALES Y MÉTODOS}

Área de estudio: Este estudio se realizó en siete vegas localizadas en el complejo montañoso "nevado de Pastos Grandes", provincia de Salta, Argentina (ecorregiones de Puna y Altoandino) entre los 3790 y $4500 \mathrm{msnm}$ (Fig. 1, Cuadro 1). El sistema hidrográfico de la

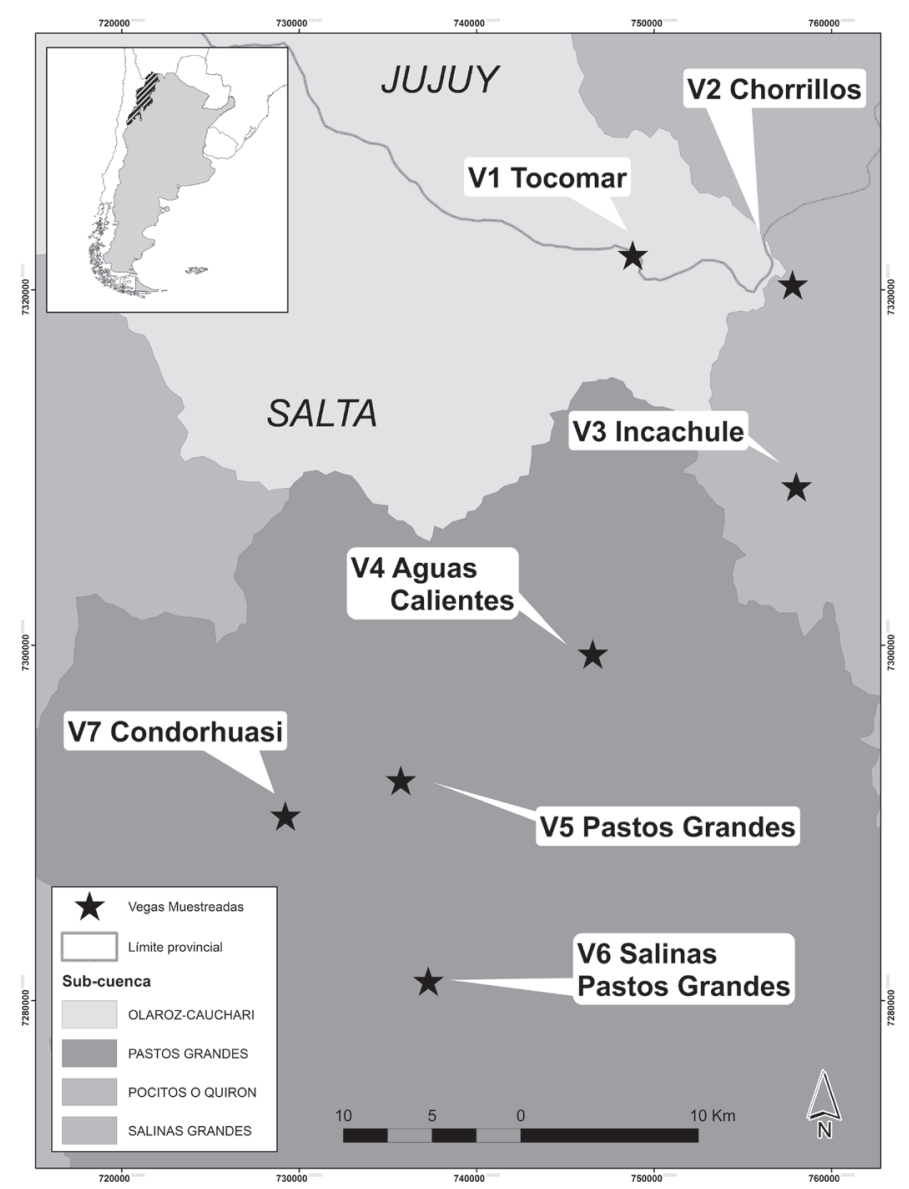

Fig. 1. Vegas muestreadas, Salta, Argentina. / Fig. 1. Sampled peatbogs, Salta, Argentina. 


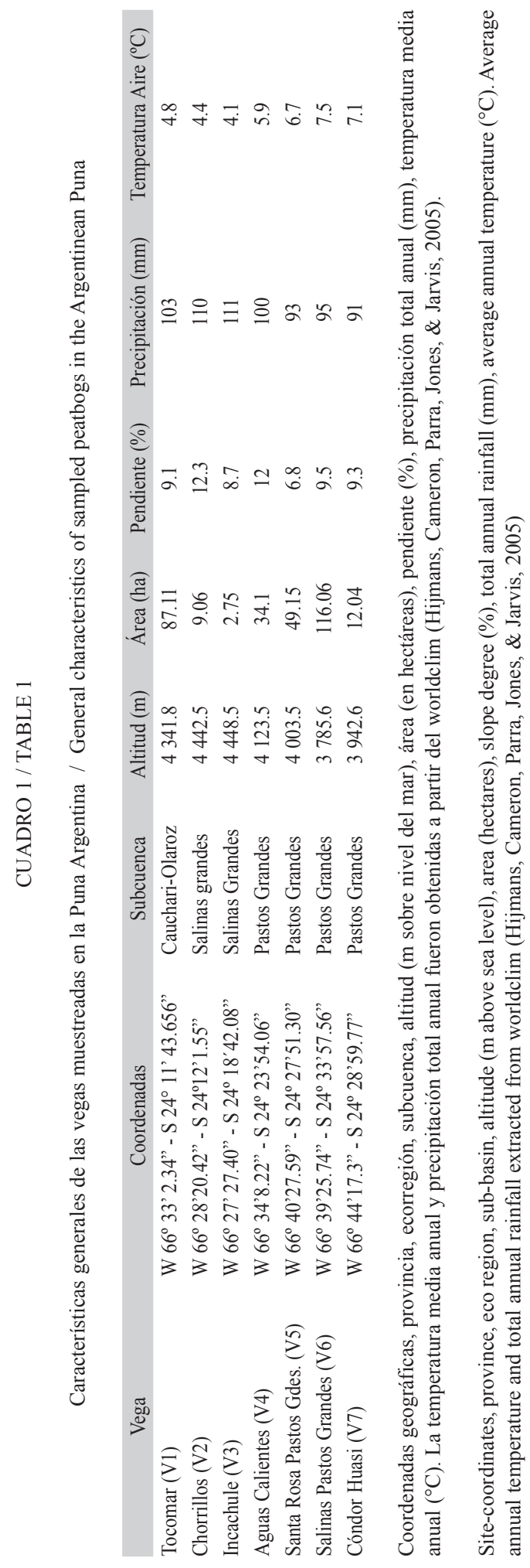

mayor parte de la Puna argentina se caracteriza por la existencia de un marcado endorreísmo, con el desarrollo de depresiones salinas que reciben los aportes de cursos fluviales de escasa magnitud y en cuyo centro suelen formarse en forma temporal o permanente cuerpos lagunares de escasa profundidad, las vegas o bofedales (Paoli, Elena, Mosciaro, Ledesma, $\&$ Noe, 2014). Las vegas son ecosistemas saturados de agua permanentemente, de elevado potencial productivo, dominados por comunidades vegetales hidromórficas, se alimentan de agua de lluvia, subterránea y de deshielo en menor medida (Ruthsatz, 2012; Alzérreca, Prieto, Laura, Luna, \& Laguna, 2001). Todas las vegas muestreadas en este estudio forman parte de la "Cuenca Cerrada de la Puna" (Paoli, Elena, Mosciaro, Ledesma, \& Noe, 2014), de las cuales Tocomar (vega 1) se encuentran en la subcuenca Cauchari-Olaroz, Chorrillos e Incachule (vegas 2 y 3) en la subcuenca Salinas Grandes y el resto (Aguas Calientes, vega 4; Pastos Grandes, vega 5; Salinas Pastos Grandes, vega 6 y Condor Huasi, vega 7) en la subcuenca de Pastos Grandes (Fig. 1, Cuadro 1). Para hacer una caracterización a escala de paisaje, Izquierdo et al., (2015) realizaron un mapeo regional de vegas de Puna y Altos Andes de Argentina del cual se obtuvo localización exacta (longitud y latitud) y área de cada vega. En base a esta información, se estimó, para cada una, la temperatura media anual y precipitación total anual a partir de worldclim (Hijmans, Cameron, Parra, Jones, \& Jarvis, 2005) (Cuadro 1). También se calculó la pendiente promedio y orientación a partir del modelo digital de elevación de SRTM $90 \mathrm{~m}$ y se determinó su altitud utilizando un equipo de GPS.

El área de las siete vegas varió entre $3 \mathrm{y}$ 116 ha (44 ha promedio), abarcó un gradiente altitudinal de $710 \mathrm{~m}$ y un gradiente latitudinal de $0.37^{\circ}$. En general, las vegas no presentaron pendientes elevadas; la pendiente promedio fue de $9.5^{\circ}$, con valores de 7 (vega 5) a $12^{\circ}$ (vegas 2 y 4). El clima de la región es frío y árido, las lluvias se concentran en los meses de verano (noviembre a marzo), con un promedio anual 
de $100 \mathrm{~mm}$, con un mínimo de 91 (vega 7) y máximo de $111 \mathrm{~mm}$ (vega 3). La temperatura promedio anual del aire fue de $5.8{ }^{\circ} \mathrm{C}$, con valores mínimos de $4.1^{\circ} \mathrm{C}$ (en vega 3 ) y máximos de $7.5^{\circ} \mathrm{C}$ (en vega 6) (Cuadro 1 ).

Se hicieron dos campañas, ambas en el período lluvioso, la primera incluyó las vegas 1 y 2 en diciembre 2013, mientras que la segunda incluyó las vegas 3-7 en febrero 2014. Tanto los muestreos de macroinvertebrados como los muestreos de agua se realizaron durante el día, entre las 10 y las $17 \mathrm{hs}$. En regiones similares de Bolivia (Wasson et al., 1989) y Ecuador (Jacobsen, 2008a) se reportaron amplitudes térmicas del agua menores durante el día (< $\left.10^{\circ} \mathrm{C}\right)$ respecto a la amplitud térmica diaria $(20$ ${ }^{\circ} \mathrm{C}$ ), por este motivo, se asumió que los horarios de muestreo en las distintas vegas no influyeron sustancialmente sobre las características físico-químicas del agua y la comunidad de macroinvertebrados.

Recolecta de muestras: Para obtener una medida cuantitativa de las densidades de macroinvertebrados en cada punto de muestreo (réplica), se utilizó la red D de $300 \mu \mathrm{m}$ de abertura de malla, y se cuantificó mediante tiempo-esfuerzo de búsqueda y recolección con una duración de 30 minutos. El número de réplicas por vega varió de 2 a 6 (25 en total) (Apéndice digital 1), eligiendo los hábitats o microambientes más disímiles entre sí. El material recolectado fue depositado en frascos de $15 \mathrm{~mL}$ y conservado en alcohol al $96 \%$. En el laboratorio, las muestras fueron separadas, cuantificadas e identificadas al nivel taxonómico más bajo que fuera posible, con la ayuda de una lupa estereoscópica y posteriormente se estimó la abundancia de cada una (Apéndice digital 1). Para su identificación se emplearon claves de macroinvertebrados sudamericanos (Domínguez \& Fernández, 2009). Las muestras se encuentran depositadas en el IBN (Instituto de Biodiversidad Neotropical, Tucumán, Argentina).

La vegetación se muestreó tanto dentro como fuera de los cauces hídricos. Para el muestreo se usó un cuadrante de $1 \mathrm{~m}^{2}$ (réplica) subdividido en 100 subcuadrantes de 0.1 x $0.1 \mathrm{~m}$. Este método ha sido utilizado para otros estudios en ambientes similares (Pauli et al., 2004). El número de cuadrantes por vega varió de 12 a 29 (117 en total). Los cuadrantes se ubicaron al azar, dentro de diferentes unidades fisonómicas-florísticas identificadas visualmente. Las plantas vasculares fueron identificadas a nivel de especie, mientras que briofitos, hongos y líquenes, solo a nivel de morfoespecie. Las especies de plantas vasculares se clasificaron según sus formas de vida, basado en bibliografía (Ruthsatz, 1978; Ramsay \& Oxley, 1997) y en conocimientos particulares. Las formas de vida consideradas fueron: 1) hierbas acuáticas (incluye plantas de aguas marginales y profundas), 2) hierbas erectas, 3) hierbas postradas (con estolones) y rastreras (sin estolones), 4) plantas en cojín o tapete, 5) graminoides en penacho, 6) graminoides dispersas, 7) graminoides cespitosas, 8) rosetas acaulescentes, y 9) vegetación no vascular (briofitos, algas y hongos). No se registraron arbustos ni helechos. Para cada vega se calcularon las siguientes variables: riqueza $=$ número de especies por vega, índice diversidad Shannon ('H) $=-$ sum $(\mathrm{Pi} * \ln (\mathrm{Pi}))$, donde $\mathrm{Pi}=$ proporción de cobertura de la especie $i$ respecto al total de cobertura (cobertura relativa de la especie $i$ ): ni/N (ni = cobertura de la especie i, $\mathrm{N}$ cobertura total de todas las especies); y equitatividad $=$ ' $\mathrm{H} / \mathrm{ln}$ riqueza, ' $\mathrm{H}$ por vega se calculó promediando los índices de los cuadrantes de $1 \mathrm{~m}^{2}$ (Cuadro 2).

En cada punto de muestreo de los macroinvertebrados, también se midió in situ, con un analizador multiparamétrico "Horiba U52" las siguientes variables: temperatura del agua, $\mathrm{pH}$, potencial óxido reducción, conductividad eléctrica, turbidez, porcentaje de oxígeno disuelto, sólidos totales disueltos y concentración de sales (Cuadro 3). Finalmente, se estimó la media de cada una de estas variables por vega.

Para describir la composición de macroinvertebrados en las vegas muestreadas se ordenaron los sitios de muestreo de cada vega $(\mathrm{n}=25$ en total) por medio de un NMDS (Non-Metrical Multidimensional Scaling). Este ordenamiento estuvo basado en una matriz de 


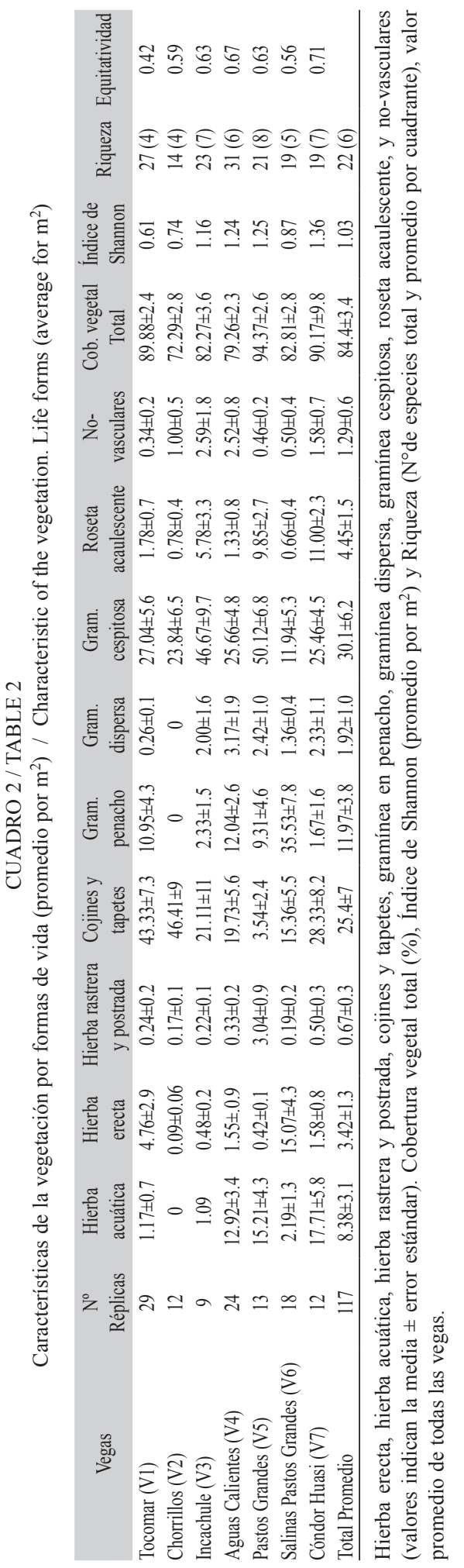

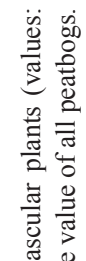

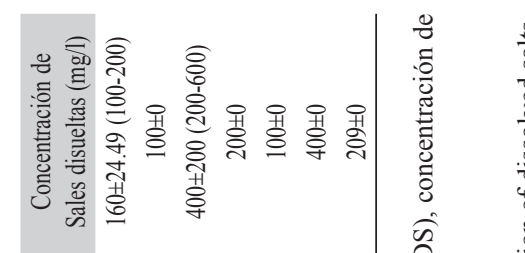

0
0

记

s.

密

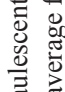

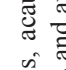

咹.

至

要

흘

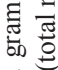

氖

일

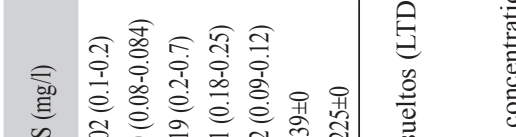

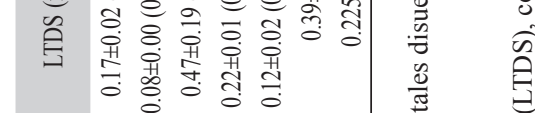

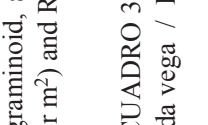

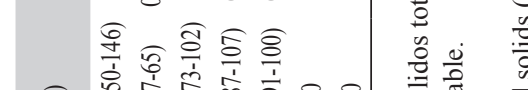

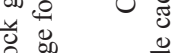

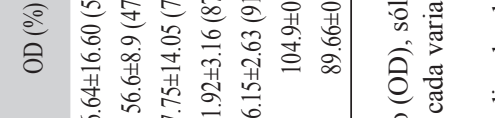

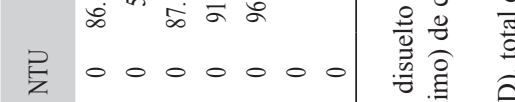

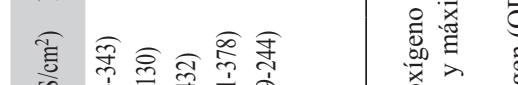

o

焉憵

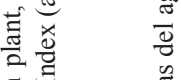

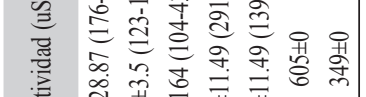

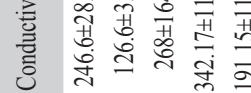

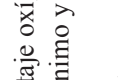

政

डิ 善

남

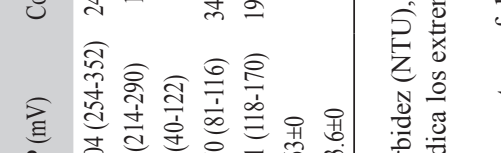

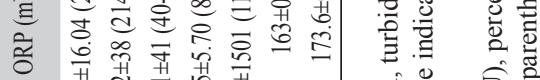

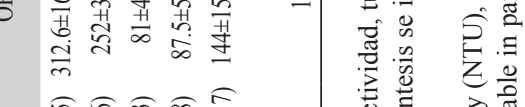

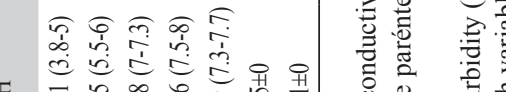

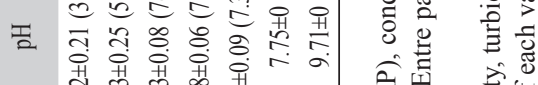

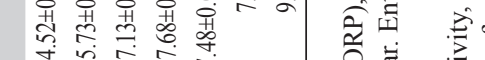

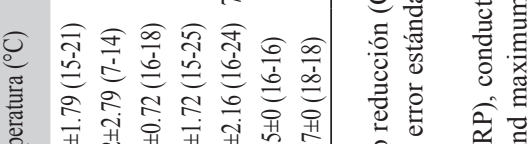

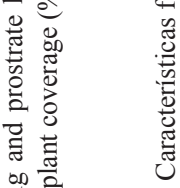

㐘

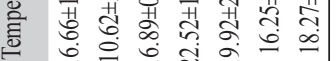

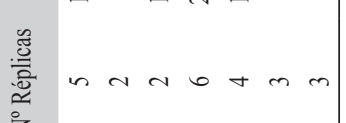

응 해

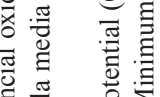

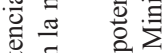

过

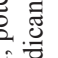

宂

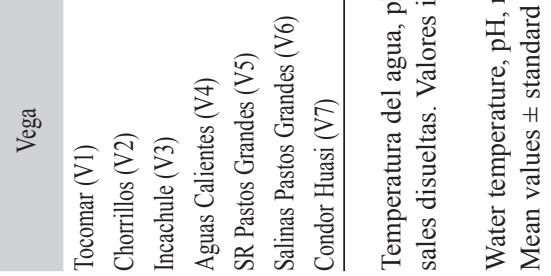


similitud de Bray Curtis entre las muestras calculadas a partir de la abundancia de macroinvertebrados. Se utilizó el modo autopiloto (modo "lento y completo") para decidir cuál dimensión era la mejor solución (McCune \& Grace, 2002). La elección de dos dimensiones se debió a que el stress final (un índice de la concordancia entre las distancias en el espacio del ordenamiento y las distancias en la matriz de Bray Curtis) fue 14.30 ( $<20$ es considerado aceptable para set de datos ecológicos) y fue diferente del azar (test de Monte Carlo $=250$ corridas con la matriz al azar, $\mathrm{p}=0.004, \mathrm{McCu}-$ ne \& Grace, 2002). La configuración espacial en los ejes del NMDS considera similares a los sitios de muestreo con una composición de macroinvertebrados comparable y como resultado se presentan cercanos en el espacio del ordenamiento. Para determinar qué especies de macroinvertebrados influyeron sobre el ordenamiento se hicieron correlaciones de Kendall tau entre la posición (score) de cada sitio de muestro sobre cada eje, con las abundancias de los macroinvertebrados. También se hicieron correlaciones de Kendall tau para determinar qué variables estuvieron relacionadas con el ordenamiento de los sitios. Dentro de las variables se incluyeron características del ambiente (longitud, latitud, altitud, temperatura promedio anual, precipitación total anual), de la vegetación circundante (índice de Shannon, cobertura, riqueza: promedio por cuadrante, y equitatividad), de las formas de vida (plantas acuáticas, hierba erecta, hierba rastrera, cojines y tapetes, graminoides penachos, graminoides dispersas, graminoides cepitosas, roseta acaulescente y no vasculares) y del agua (temperatura, $\mathrm{pH}$, conductividad, porcentaje de oxígeno disuelto, potencial óxido reducción, turbidez, concentración de sales, sólidos totales disueltos). A posteriori, para las pruebas múltiples, se utilizaron correcciones de tasas de descubrimientos falsos (FDR, por su nombre en inglés False Discovery Rate) para limitar la aceptación de hipótesis basadas sólo en azar. FDR es una corrección que examina el valor de $\mathrm{P}$ individual de cada prueba y considera el número (proporción) de pruebas que sean significativas (Benjamini \& Hochberg, 1995). Se calculó el coeficiente de determinación (porcentaje de varianza representada por cada eje) entre las distancias originales en la matriz de Bray Curtis y las distancias en el espacio del ordenamiento de cada cuadrante sobre cada uno de los ejes para tener una estimación de la varianza explicada por cada eje (McCune \& Grace, 2002). Finalmente, se realizó un análisis de correlación de Kendall tau no paramétrico entre las variables químicas del agua.

\section{RESULTADOS}

Descripción general de la comunidad de macroinvertebrados: Se recolectaron 3131 individuos de macroinvertebrados acuáticos. Se identificaron 25 taxa pertenecientes a 22 familias y 24 géneros. Las familias que presentaron las mayores abundancias fueron Baetidae (Ephemeroptera), Simuliidae (Diptera) y Hyalellidae (Amphipoda) mientras que Dytiscidae, Hydrophilidae y Staphylinidae (Coleoptera) presentaron los valores más bajos (Apéndice 1, digital). Claramente los insectos fueron los dominantes en la composición de la comunidad de macroinvertebrados. El orden Coleoptera fue el mejor representado en cuanto a familias consideradas, ya que se encontraron cinco diferentes.

Descripción general de la vegetación: Se registraron un total de 62 especies de plantas vasculares, pertenecientes a 20 familias (Apéndice digital 2). Las especies más abundantes en términos de cobertura (\%) fueron: Eleocharis atacamensis, E. pseudoalbibracteata, Juncus balticus, Limosella australis, Oxychloe andina, y Zameioscirpus atacamensis. Mientras que las más ampliamente distribuidas: Gentiana prostrata, Halerpestes exilis, Deyeuxia curvula, Lobelia oligophylla, Oxychloe andina, Plantago tubulosa y Werneria pygmaea var. apiculata presentes en al menos seis de las siete vegas. El porcentaje de cobertura promedio por vega, fue de $84.4 \pm 2.8$, estimado a partir del promedio de la cobertura de los cuadrantes de $1 \mathrm{~m}^{2}$, la vega 5 presentó la cobertura máxima $(94.4 \%)$ y la vega 2 la mínima (72.3\%, Cuadro 2$)$. La 
riqueza total por vega varió de 14 (vega 2) a 31 (vega 4) especies de plantas vasculares; la riqueza promedio por $\mathrm{m}^{2}$ varió entre 4 (vega 1 y 2) y 8 (vega 5) especies, registrándose hasta 13 especies por $\mathrm{m}^{2}$ en las vegas 5 y 6 . La diversidad ' $\mathrm{H}$ promedio fue de $1.03( \pm 0.30)$ siendo mayor en la vega 7 con 1.36 y menor en la vega 1 con 0.61 (Cuadro 2).

La forma de vida más abundante fueron las graminoides cespitosas, con un promedio de 30 $\%$ (hasta $50 \%$ en la vega 5 ), seguida por cojines y tapetes con $25 \%$ promedio (hasta $46 \%$ en vega 2). En las vegas 1, 2 y 7 la forma de vida predominante fueron los cojines y tapetes, en las vegas $3,4,5$ predominaron las graminoides cespitosas, y en la vega 6 las graminoides en penacho. Las formas de vida menos abundantes fueron las hierbas erectas para las vegas 2 y 5 y las hierbas rastreras para las otras vegas (vegas 1, 3, 4, 6 y 7, Cuadro 2).

Descripción general de las características físico-químicas del agua: La temperatura del agua fue variable entre las vegas, osciló entre los $10{ }^{\circ} \mathrm{C}$ (vega 2 ) y $22^{\circ} \mathrm{C}$ (vega 4 ), durante el día. La conductividad presentó valores mínimos de $126 \mu \mathrm{S} / \mathrm{cm}$ (vega 2) y máximo de 605 $\mu \mathrm{S} / \mathrm{cm}$ en la vega 6 . Patrón coincidente con la concentración de sales; valores mínimos de 100 $\mathrm{mg} / \mathrm{L}$ en vegas 2 y 5 , y valores máximos de 400 $\mathrm{mg} / \mathrm{L}$ en vegas 3 y 6 (esperable para la vega asociada a una salina, vega 6). Los sólidos totales disueltos mostraron valores similares, oscilaron entre valores mínimos de $0.08-0.12$ (vega 2 y 5) y valores máximos de $0.39-0.47 \mathrm{mg} / \mathrm{L}$ (vega 3 y 6 , Cuadro 3). El pH también presentó variaciones entre las vegas, osciló entre neutro o levemente alcalino hasta ácido. La vega 7 fue la más alcalina con un valor de 9, mientras que la vega 1 fue la más ácida con un valor de 3.8. La turbidez siempre estuvo por debajo de cero mientras que el porcentaje de oxígeno disuelto presentó valores por encima del $50 \%$, hasta un $104 \%$ en la vega 6.

Distribución espacial de los macroinvertebrados y su relación con las características ambientales, florísticas y físico-químicas del agua: Los sitios de muestreo de las diferentes vegas tendieron a segregarse a lo largo de dos ejes del NMDS basado en la composición de macroinvertebrados (varianza representada por el eje $1=58.4 \%$ y varianza representada por el eje $2=22.6 \%$, varianza total representada por ambos ejes $=81.0 \%$, Fig. 2). La abundancia de ciertos taxa, tales como Andesiops peruvianus, Massartellopsis (Ephemeroptera), Austrelmis (larva y adulto, Elmidae, Coleoptera), Hydroptilidae (Coleoptera), Simuliidae (Diptera), Hydrobiosidae y Odontoceridae (Trichoptera) y

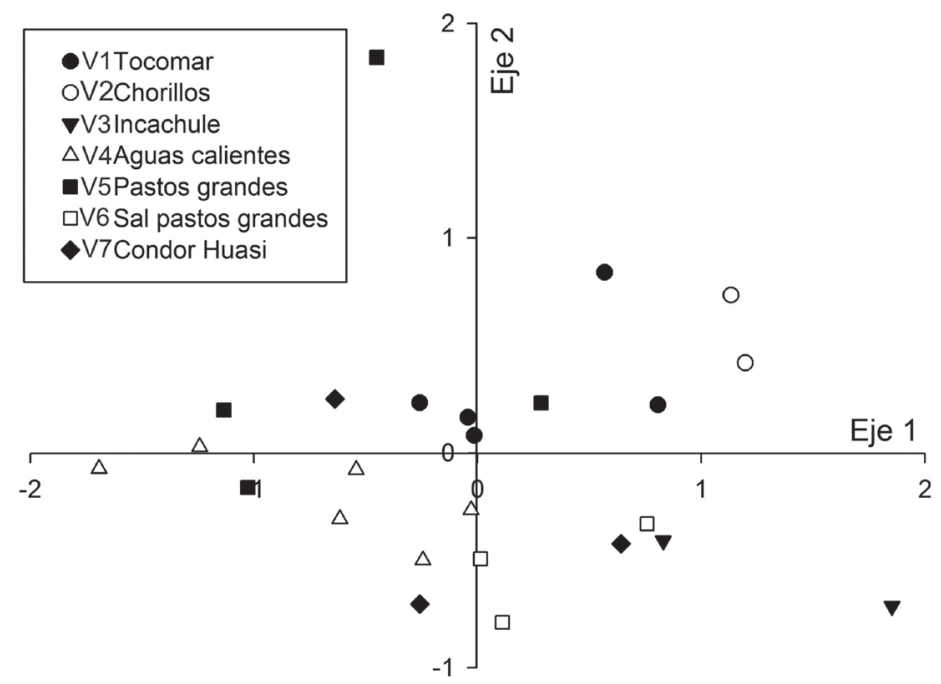

Fig. 2. Diagrama de ordenamiento de los sitios de muestreo de las diferentes vegas a lo largo de dos ejes del NMDS. Fig. 2. Ordination of the sampling sites of each peatbogs along NMDS axes. 
Glossiphonidae (Hirudinea), se incrementaron hacia el lado negativo del eje 1, mientras que Limnophora y Orthocladiinae (Diptera) incrementaron su abundancia hacia el lado positivo del eje 1 (Cuadro 4). Otros taxa aumentaron su abundancia hacia el lado negativo del eje 2, como Protallagma titicacae (Odonata), Hydrophilidae (Coleoptera), Hyalella (Crustacea) y Bivalvia (Molusco, Cuadro 4) mientras que Podonominae (Diptera) y Limnephilidae (Trichoptera) lo hicieron hacia el lado positivo.

A partir del ordenamiento de los distintos sitios de muestreo y las variables ambientales,

\section{CUADRO 4}

Coeficientes de correlación de Kendal entre la abundancia de especies de macroinvertebrados y los valores de las unidades de muestreo sobre los ejes del ordenamiento NMDS

TABLE 4

Kendal correlation between macroinvertebrates and sampling units along the NMDS axes

\begin{tabular}{lcc} 
& Eje 1 & Eje 2 \\
Insectos & & \\
Protallagma titicacae & -0.14 & $-0.40^{* *}$ \\
Claudioperla rosalesi & -0.09 & -0.08 \\
Andesiops peruvianus & $-0.75^{* * *}$ & -0.05 \\
Massartellopsis & $-0.52^{* * *}$ & -0.05 \\
Hydrophilidae & -0.07 & $-0.32^{*}$ \\
Austrelmis (larva) & $-0.45^{* * *}$ & -0.27 \\
Austrelmis (adulto) & $-0.58^{* * *}$ & -0.04 \\
Staphylinidae & -0.16 & -0.16 \\
Dytiscidae & 0.28 & -0.26 \\
Simuliidae & $-0.73^{* * *}$ & -0.06 \\
Chironomidae & 0.09 & 0.10 \\
Podonominae & 0.25 & $0.34 *$ \\
Ortocladiinae & $0.32^{*}$ & -0.02 \\
Limnophora & $0.53^{* * *}$ & 0.10 \\
Hydrobiosidae & $-0.37^{*}$ & -0.11 \\
Limnephilidae & -0.07 & $0.32^{*}$ \\
Odontoceridae & $-0.31^{*}$ & -0.04 \\
Hydroptilidae & $-0.39^{* *}$ & -0.01 \\
Trichocorixa & 0.10 & -0.12 \\
No insectos & & \\
Hyalella & 0.12 & $-0.48^{* * *}$ \\
Gasteropoda & -0.12 \\
Bivalvia & -0.03 & $-0.47 * *$ \\
Oligochaeta & -0.11 & 0.26 \\
Acari & -0.28 & 0.21 \\
Glossiphonidae & $-0.33^{*}$ & 0.05 \\
\hline & &
\end{tabular}

${ }^{*} \mathrm{p}<0.05, * * \mathrm{p}<0.01, * * * \mathrm{p}<0.001$. solamente la longitud mostró una asociación significativa negativa con el eje 1 , indicando que hacia el lado negativo del eje 1 , se encuentran las vegas ubicadas más al oeste y más al sur.

Dentro de los distintos tipos de formas de vida las graminoides dispersas, las hierbas rastreras y las plantas acuáticas fueron más abundantes hacia el lado negativo del eje 1 del ordenamiento (Cuadro 5), ubicando a las

\section{CUADRO 5}

Coeficientes de correlación de Kendall entre las características ambientales, de vegetación circundante y físico químicas del agua con cada sitio de muestreo y sus valores sobre los ejes 1 y 2 del ordenamiento de escalamiento multidimensional no métrico (NMDS)

TABLE 5

Kendal correlation between environmental, vegetation and physico-chemical characteristics of water and each sampling site and its values in the NMDS axes

\begin{tabular}{|c|c|c|c|}
\hline & Características & Eje 1 & Eje 2 \\
\hline \multirow{5}{*}{ 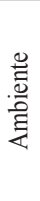 } & Longitud & $-0.40^{*}$ & -0.13 \\
\hline & Latitud & -0.12 & -0.30 \\
\hline & Altitud & 0.24 & 0.26 \\
\hline & Precipitación total anual (mm) & 0.36 & 0.15 \\
\hline & Temperatura media anual $\left({ }^{\circ} \mathrm{C}\right)$ & -0.27 & 0.26 \\
\hline \multirow{11}{*}{ 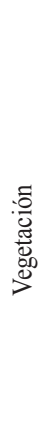 } & Cojines y tapetes & 0.30 & 0.21 \\
\hline & Hierba erecta & 0.07 & -0.21 \\
\hline & Roseta acaulescente & -0.11 & 0.14 \\
\hline & Gramínea dispersa & $-0.49 * *$ & -0.21 \\
\hline & Gramínea cespitosa & -0.12 & 0.25 \\
\hline & Gramínea penacho & -0.20 & -0.32 \\
\hline & No-vasculares & -0.03 & -0.37 \\
\hline & Hierba rastrera y postrada & $-0.53 * *$ & 0.07 \\
\hline & Acuática & $-0.49 * *$ & -0.08 \\
\hline & Índice de Shannon & -0.30 & -0.11 \\
\hline & Riqueza & -028 & -0.17 \\
\hline \multirow{9}{*}{ 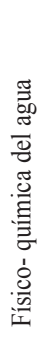 } & Equitatividad & -0.27 & -0.23 \\
\hline & Temperatura agua $\left({ }^{\circ} \mathrm{C}\right)$ & $-0.42 *$ & -0.12 \\
\hline & $\mathrm{pH}$ & -0.23 & -0.32 \\
\hline & ORPmV & 0.18 & 0.31 \\
\hline & $\mathrm{uS} / \mathrm{cm}$ & -0.10 & $-0.44 *$ \\
\hline & NTU/Turbidez & -0.01 & -0.16 \\
\hline & $\%$ DO & -0.03 & -0.18 \\
\hline & $\mathrm{S} / \mathrm{LTDS} /$ Sólidos totales disueltos & 0.05 & $-0.59 * * *$ \\
\hline & Concentración de sales disueltas & 0.14 & $-0.57 * * *$ \\
\hline
\end{tabular}

${ }^{*} \mathrm{p}<0.05,{ }^{* *} \mathrm{p}<0.01, * * * \mathrm{p}<0.001$. 
CUADRO 6

Coeficiente de correlación de Kendall no paramétrico entre las variables químicas del agua

TABLE 6

Non-parametric Kendal correlations among physico- chemical characteristics of water

\begin{tabular}{|c|c|c|c|c|c|c|c|c|}
\hline & T. Agua & $\mathrm{pH}$ & ORP mV & $\mathrm{uS} / \mathrm{cm}$ & NTU/ Turbidez & $\% \mathrm{OD}$ & S/LTDS & Concentración de sales \\
\hline T. Agua & 1.00 & -- & -- & -- & -- & -- & -- & -- \\
\hline $\mathrm{pH}$ & 0.21 & 1.00 & -- & -- & -- & -- & -- & -- \\
\hline ORPmV & $-0.32 *$ & $-0.52 *$ & 1.00 & -- & -- & -- & -- & -- \\
\hline $\mathrm{uS} / \mathrm{cm}$ & 0.20 & $0.33^{*}$ & -0.25 & 1.00 & -- & -- & -- & -- \\
\hline NTU/Turbidez & $-0.32 *$ & 0.02 & 0.19 & 0.12 & 1.00 & -- & -- & -- \\
\hline$\% \mathrm{OD}$ & -0.17 & $0.47^{*}$ & -0.08 & 0.14 & 0.19 & 1.00 & -- & -- \\
\hline S/LTDS & 0.15 & $0.37 *$ & $-0.35^{*}$ & $0.81^{*}$ & 0.09 & 0.20 & 1.00 & -- \\
\hline Concentración de sales & -0.01 & 0.24 & -0.19 & $0.64 *$ & 0.22 & 0.26 & $0.80 *$ & 1.00 \\
\hline
\end{tabular}

$* \mathrm{p}<0.05$

vegas 4,5 y 7 de este lado del eje. Dentro de las graminoides dispersas se incluyen especies de Poacea como Poa parviceps, P. superata y Puccinellia frigida y Cyperaceae como Carex macloviana. En el grupo de las hierbas rastreras y postradas se incluyen a Astragalus bustillosii, A. tacorensis, A. uniflorus, Cotula mexicana y Halerpes exilis, cabe destacar que se las agrupó dentro de la misma forma de vida por su escasa cobertura. Dentro de las plantas acuáticas se encuentran varias familias representadas por Lilaeopsis macloviana (Apiaceae), Limosella australis (Plantaginaceae), Lobelia oligophylla (Campanulaceae), Mimulus luteus (Phrymaceae), Myriophyllum quitense (Halarogaceae) y Stuckenia striata (Potamogenaceae) (Apéndice 2, digital).

A partir del NMDS, las vegas con mayor temperatura de agua se ubicaron en la parte negativa del eje 1 (vegas más al oeste), mientras que las vegas con mayor conductividad, sólidos totales disueltos, y concentración de sales en el agua, se ubicaron en la parte negativa del eje 2 (Cuadro 5). El pH, la turbidez y el porcentaje de oxígeno disuelto no se relacionaron con los ejes del ordenamiento (Cuadro 6).

\section{DISCUSION}

Las comunidades de macroinvertebrados observadas en el presente estudio son similares en cuanto a su composición taxonómica a las reportadas en otros cauces hídricos andinos de América del Sur. Tal es el caso de las familias Baetidae, Elmidae, Simuliidae, Chironomidae, Gripopterygidae, Hyalellidae que fueron recolectadas previamente en los Andes de Colombia (Muñoz-Quesada, 2004; Zuñiga, Molineri, \& Domínguez, 2004), Ecuador (Jacobsen, 2008 a y b; Maldonado et al., 2011) y Bolivia (Jacobsen \& Marín, 2008; Molina, Gibon, Pinto, \& Rosales, 2008), incluso compartiendo especies como Andesiops peruvianus y Meridialaris tintinnabula (Molina et al., 2008).

Las formas de vida de plantas como gramíneas dispersas, hierbas rastreras y postradas (que en muchos casos ingresan al cauce de agua de las vegas) y plantas acuáticas fueron más abundante en las vegas 4, 5 y 7 (ubicadas hacia el lado negativo del eje 1) y estuvieron asociadas con ciertos taxa de macroinvertebrados que pertenecen a distintos grupos funcionales tróficos como colectores-filtradores (Simuliidae), colectores-recolectores Massartellopsis, y raspadores (Austrelmis, Hydroptilidae y Andesiops peruvianus) (Jacobsen, 2008a; Reynaga \& Dos Santos, 2012). Esto puede explicarse a partir de que estas plantas generan una mayor disponibilidad de recursos tanto alimenticio como de refugio (Esteves, 1998; Flory \& Milner, 1999; Moya et al., 2009). Conjuntamente con el aumento en la abundancia 
de estos macroinvertebrados, se incrementó la abundancia de Hydrobiosidae y Glossiphonidae, ambos considerados depredadores. Respecto a las formas de vida de las plantas, anteriormente mencionadas (gramíneas dispersas, hierbas rastreras y postradas, y plantas acuáticas), resultaron ser las menos representadas en las vegas, siendo apenas más abundantes en las vegas mencionadas (4, 5 y 7 ).

Con respecto a las características físicoquímicas del agua, las variables de conductividad, sólidos totales disueltos y concentración de sales fueron más elevados en la vega 6 (ubicada hacia el lado negativo del eje 2 del ordenamiento) donde estuvieron asociados los Bivalvia (colector-filtrador) y Hyallela (colector-recolector). En este último caso, Acosta \& Prat (2011) realizaron estudios de la dieta alimentaria en una cuenca altoandina de Perú a $3800 \mathrm{~m}$ y encontraron, aparte de la materia particulada gruesa y fina, una proporción considerable de mineral (precipitado de carbonato de calcio). Esto es consistente con los resultados aquí obtenidos, dado que aumentó la abundancia de estos individuos en la medida que aumentó la disponibilidad de carbonato de calcio. En el caso de Bivalvia la disponibilidad de sales puede estar relacionada con la producción de su conchilla. White, Chejlava, Fried, \& Sherma (2007) concluyeron que en las aguas con mayor concentración de carbonato de calcio se encuentran las especies de Mollusca con mayor porcentaje de carbonato de calcio en sus conchillas.

En general, las vegas más al oeste y más al sur (vegas 4, 5, 6 y 7) presentaron una mayor asociación en cuanto a los taxa de plantas y macroinvertebrados encontrados. Esto podría estar relacionado con el hecho de que forman parte de la misma subcuenca (Pastos Grandes) a diferencia de las vegas que están más al este (vegas 1, 2 y 3) que pertenecen a la subcuenca Olaroz-Cauchari y Salinas Grandes, respectivamente, y que tienen una conexión más directa con la cordillera oriental/borde oriental de la Puna. Cabe aclarar que las diferencias marcadas de algunas variables en la vega 2 , como ser una menor riqueza específica de plantas y la ausencia de gramíneas en penacho y gramíneas dispersas, se atribuyen a que fue atravesada por las vías del tren en la primera mitad del siglo XX y se secó temporalmente.

Una de las características más distintivas de los cauces hídricos andinos de altura, es la escasez de trituradores, tanto en especies como en abundancia (Dudgeon, 1989; 2000). Esto puede deberse a que la temperatura del agua es mayor a la temperatura del aire, lo que ocasiona un incremento de la actividad microbiana y la tasa de descomposición de la hojarasca, con lo cual el detrito no está disponible para los trituradores (Irons, Oswood, Stout, \& Pringle, 1994). Con la posible excepción de Hyallela, en este trabajo no fueron reportados trituradores. Estos crustáceos presentan un hábito alimenticio discutido, ya que para algunos autores son trituradores (Cummins, Merrit, \& Andrade, 2005), o trituradores facultativos (Saigo, Marchese, \& Montalvo, 2009), o colector-recolectores (Saigo et al., 2009), o raspadores, o hasta incluso depredadores (Wantzen \& Wagner, 2006). Por otra parte, los colectores-filtradores son ocasionalmente muy abundantes pero generalmente no son dominantes (Encalada, 1997) en los cauces tropicales de altura. Este es el caso de Simuliidae que estuvo presente en cinco de las siete vegas, pero nunca fue el taxón dominante en las comunidades encontradas.

La altitud es considerada como una de las variables con mayor importancia para la biota de los ríos (Vannote, Minshall, Cummins, Sedell, \& Cushing, 1980; Rahbeck, 1995). La altitud refleja la variabilidad de factores como la presión atmosférica, la concentración de oxígeno, la vegetación riparia, los hábitats existentes dentro del río y la calidad y cantidad de la materia orgánica (Vinson \& Hawkins, 1998; Jacobsen, 2003; Jacobsen, Rostgaard, \& Vasconez, 2003). La altitud también afecta la temperatura del agua y del aire; la temperatura tiende a disminuir en la medida que se incrementa la altura. Sin embargo, no es la única característica que influye, también intervienen la radiación solar, la infiltración y la pérdida de calor por evapotranspiración (Ward, 1985; Walling \& Webb, 1992; Cassie, 2006). Los 
cauces hídricos a menor altura (2 500-3 000 msnm) tienden a ser más fríos que el aire, mientras que a mayor altura tienden a ser más cálidos que el aire (Jacobsen, 2008a). Esto se debe principalmente a la mayor densidad de vegetación, en altitudes más bajas, seguido de una pérdida de calor por evapotranspiración. Coincidente con los resultados aquí obtenidos, la temperatura del agua fue siempre mayor que la del aire teniendo en cuenta que el rango altitudinal estuvo por encima de los $3800 \mathrm{~m}$, y no mostró un patrón claro de disminución con la altitud, viéndose afectada mayormente por la radiación solar y la menor cobertura de la vegetación circundante. La temperatura del agua registrada fue bastante elevada, comparada con otros estudios de vegas (Molina et al., 2008). Las vegas ubicadas hacia el oeste, y de menor altitud (subcuenca Pastos Grandes) presentaron temperaturas del agua más elevadas (promedio $19{ }^{\circ} \mathrm{C}$ ) respecto a las ubicadas hacia el este y de mayor altitud (promedio 14.7 ${ }^{\circ} \mathrm{C}$ ), como se esperaría con el patrón altitudinal. Por otra parte, la altitud también afecta a diferentes grupos de plantas y animales acuáticos (Rahbek, 1995, 2005), incluso terrestres (Mani, 1968; Janzen et al., 1976; McCoy, 1990; Brehm, Colwell, \& Kluge, 2007), disminuyendo la riqueza específica a medida que aumenta el gradiente altitudinal. Sin embargo, en el caso de los macroinvertebrados este patrón no parece manifestarse tan claramente (Jacobsen, 2008a, b). Esto concuerda con lo obtenido en este trabajo, en donde la altitud no se correlacionó con la abundancia de los macroinvertebrados, aunque también podría deberse a que la variación altitudinal fue de $710 \mathrm{~m}$. La riqueza de plantas tampoco mostró este patrón con la altura, pero sí se observó un patrón coincidente con la temperatura del agua; la vega 4 , con el mayor número de especies (31), registró una temperatura del agua de $22{ }^{\circ} \mathrm{C}$ y la vega 2 con el menor número de especies (14), registró una temperatura de $10{ }^{\circ} \mathrm{C}$. Si bien el $\mathrm{pH}$ no se asoció a ninguno de los ejes, tuvo una relación positiva con la conductividad (tau $=$ 0.33 ) es decir que las vegas con valores más altos de conductividad tendieron a presentar
pH básicos. Todos los sitios de la vega Tocomar (vega 1) presentaron un $\mathrm{pH}$ ácido entre $3.8 \mathrm{y}$ 4.5. Ciertos estudios demostraron que un $\mathrm{pH}$ menor a 4 influye negativamente en la abundancia de ciertos taxa particularmente sensibles a la acidificación del agua (Tripole, Vallania, \& Corigliano, 2008), tal es el caso de los moluscos. En esta vega no se reportó la presencia de Bivalvia ni de Gasteropoda. La conductividad, los sólidos totales disueltos y la concentración de sales disminuyeron con la altura. En la cuenca alta del río Chama, Venezuela, se reportaron características similares (Segnini \& Chacón, 2005). Sin embargo, este patrón está lejos de ser una regla en los cauces hídricos de altura. Maldonado \& Goitia (2003) en Cochabamba (Bolivia) y Monaghan et al. (2000) en los Andes de Ecuador concluyeron que la geografía regional explica mejor las variaciones químicas del agua de los ríos que la altitud o las alteraciones humanas sobre las cuencas.

Si bien en el presente trabajo no se evaluaron aspectos relacionados a los impactos antrópicos que sufren las vegas, resulta relevante remarcar que estos ecosistemas podrían ser muy sensibles al cambio climático (Beniston, Diaz, \& Bradley, 1997) y patrones de uso del suelo como la ganadería (Reboratti, 2006) y la minería (Seijmonsbegen, Sevink, Cammeratt, \& Recharte, 2010). Conocer cómo responden las comunidades de macroinvertebrados a posibles diferencias en las características físicoquímicas, y evaluar la asociación de estos cambios al régimen de disturbio del sistema, podría ser información de relevancia para ajustar Índices Biológicos para evaluar la calidad de agua (Gibson, Barbour, Stribling, Gerritsen, \& Karr, 1996), aplicación para la cual los macroinvertebrados son un grupo ampliamente usado (Fernández, Domínguez, Romero, \& Cuezzo, 2006).

A partir de los resultados obtenidos se evidencia que los macroinvertebrados presentaron patrones espaciales de diversidad y composición, siendo más importante su relación con la vegetación circundante y en segundo término con las características físico-químicas del agua en siete vegas de la Puna argentina. 
A su vez, las formas de vida de plantas menos abundantes y distribuidas más localmente, serían mejores indicadores de determinados ambientes y estarían asociadas a la presencia de ciertos macroinvertebrados. Por último, la altitud no pareció afectar la riqueza específica de los macroinvertebrados como tampoco la de las plantas.

\section{AGRADECIMIENTOS}

Los autores agradecen a los cuatro revisores, a Daisy Arroyo Mora y a Alonso Prendas Vega cuyos comentarios y observaciones contribuyeron a mejorar este manuscrito. A Fátima Romero por los aportes y sugerencias en la primera etapa del trabajo. Los autores también agradecen a CONICET (Consejo Nacional de Investigaciones Científicas y Técnicas), por el apoyo económico. Este estudio fue financiado por los proyectos: PICT 1565, PIP 0330, PIUNT cn-G516, FOCA-Galicia y Booster Rufford Foundation.

\section{RESUMEN}

Los macroinvertebrados son un componente vital en los ecosistemas acuáticos, procesan materia orgánica y sirven de alimento para otros organismos como los peces o anfibios. En los sistemas altoandinos, y más específicamente en las vegas o bofedales de Argentina, el conocimiento de la diversidad acuática es escaso o nulo, lo que ha dificultado la interpretación de patrones espaciales a escalas regionales. Típicamente estos ecosistemas se sitúan entre los 3200 y 5200 m.s.n.m. en zonas con precipitaciones por debajo de los $300 \mathrm{~mm}$ anuales. En este trabajo se realizó la primera descripción cuantitativa de los patrones espaciales de diversidad y composición de macroinvertebrados acuáticos en siete vegas de la Puna Argentina, y su relación con las características del ambiente, de la vegetación circundante y las características físico-químicas del agua. Se recolectaron 3131 individuos de macroinvertebrados acuáticos, los cuales fueron identificados en 25 taxa que pertenecen a 22 familias y 24 géneros. Entre las plantas vasculares se registraron un total de 62 especies, correspondiente a 20 familias, las graminoides cespitosas fueron la forma de vida más abundante seguida por plantas en cojín o tapete. A partir de un análisis estadístico de ordenamiento NMDS (Non-Metrical Multidimensional Scaling) se segregaron los sitios de muestreo de cada vega. Las vegas ubicadas más al oeste y al sur y con mayor temperatura del agua se agruparon sobre el eje 1 , mientras que las vegas con mayor conductividad, sólidos totales disueltos, y concentración de sales en el agua lo hicieron sobre el eje 2. La temperatura del agua fue siempre mayor que la del aire, y no mostró un patrón claro de disminución con la altitud. La altitud no se correlacionó con la abundancia de los macroinvertebrados ni con la riqueza de las plantas. Las formas de vida de plantas como gramíneas dispersas, hierbas rastreras y postradas (que en muchos casos ingresan al cauce de agua) y plantas acuáticas fueron más abundantes en las vegas 4, 5 y 7 y estuvieron asociadas con ciertos taxa de macroinvertebrados que pertenecen a distintos grupos funcionales tróficos como colectores- filtradores, colectores- recolectores y raspadores. Finalmente, las variables de conductividad, sólidos totales disueltos y concentración de sales fueron más elevadas en la vega 6, asociadas a Bivalvia (colector-filtrador) y Hyalella (colector-recolector). A partir de estos resultados se evidencia que los macroinvertebrados presentaron patrones espaciales de diversidad y composición, siendo en primer lugar más importante su relación con la vegetación circundante y en segundo término con las características físico-químicas del agua en siete vegas de la Puna argentina.

Palabras clave: macroinvertebrados, vegas, vegetación, ecosistemas acuáticos, puna, neotropical.

\section{REFERENCIAS}

Acosta, R., \& Prat, N. (2011). Trophic ecology of Hyalella sp. (Crustacea: Amphipoda) in a high Andes headwater river with travertine deposits. International Review of Hydrobiology, 96(3), 274-285.

Alzérreca, H., Prieto, G., Laura, J., Luna, D., \& Laguna, S. (2001). Características y distribución de los bofedales en el Ámbito Boliviano (Informe de Consultoría). La Paz, Bolivia: Asociación Integral de Ganaderos en Camélidos de los Andes Altos (AIGACAA), Autoridad Binacional del Lago Titicaca (ALT), Gerencia del Proyecto de Biodiversidad.

Beniston, M., Diaz, H. F., \& Bradley, R. S. (1997). Climatic change at high elevation sites: an overview. Climatic Change, 36(3-4), 233-251.

Benjamini, Y., \& Hochberg, Y. (1995). Controlling the false discovery rate: a practical and powerful approach to multiple testing. Journal of the Royal Statistical Society B, 57, 289-300.

Brehm, G., Colwell, R. K., \& Kluge, J. (2007). The role of environmental and mid-domain effect on moth species richness along a tropical elevational gradient. Global Ecology and Biogeography, 16, 205-219.

Cassie, D. (2006). The thermal regime of rivers: a review. Freshwater Biology, 51, 1389-1406.

Cummins, K. W., Merrit, R. W., \& Andrade, P. C. N. (2005). The use of invertebrate funcional groups to 
characterize ecosystem attributes in selected streams and rivers in south Brazil. Studies on Neotropical Fauna and Environment, 40(1), 69-89.

Domínguez, E., \& Fernández, H. R. (Eds.). (2009). Macroinvertebrados bentónicos sudamericanos. Sistemática y Biología. Tucumán, Argentina: Fundación Miguel Lillo.

Domínguez, E., Molineri, C., Pescador, M. L., Hubbard, M. D., \& Nieto, C. (2006). Ephemeroptera of South America. In J. Adis, J. R. Arias, K. Wantzen, \& G. Rueda (Eds.). Aquatic Biodiversity of Latin America (p. 332). Sofia-Moscow: Pensoft Press.

Donato, J. (2001). Fitoplancton de los lagos andinos del norte de Sudamérica (Colombia). Composición física y factores de distribución. Bogotá: Academia Colombiana de Ciencias Exactas, Físicas y Naturales, y Editora Guadalupe.

Dudgeon, D. (1989). The influence of riparian vegetation on the functional organization of four Hong Kong stream communities. Hydrobiologia, 179(3), 183-194.

Dudgeon, D. (2000). The ecology of tropical Asian rivers and streams in relation to biodiversity conservation. Annual Review of Ecology and Systematics, 31, 239-263.

Edmunds, G. F. Jr., Jensen, S. L., \& Berner, L. (1976). Mayflies of North and Central America. Minneaapolis: University of Minnesota Press.

Encalada, A. (1997). Diversidad y abundancia de macroinvertebrados en relación a factores físico-químicos $y$ de fuentes de alimento en dos tipos de rios de los páramos del Ecuador (Tesis de Licenciatura). Pontifica Universidad Católica del Ecuador, Quito.

Esteves, F. A. (1998). Fundamentos de limnologia (2. Ed). Rio de Janeiro: Interciência.

Fernández, H. R., Domínguez, E., Romero, F., \& Cuezzo, M. G. (2006). La calidad del agua y la bioindicación en los ríos de montaña del Noroeste Argentino. Serie Conservación de la Naturaleza 16. Tucumán: Fundación Miguel Lillo.

Flory, E., \& Milner, A. (1999). Influence of riparian vegetation on invertebrate assemblages in a recent formed stream in Glacier Bay National Park, Alaska. Journal of the North American Benthological Society, 18, 261-273.

Gibson, G. R., Barbour, M. T., Stribling, J. B., Gerritsen, J., \& Karr, J. R. (1996). Biological Criteria and Technical Guidance for Streams and Small Rivers. EPA 822-B-96-001. US: USEPA, Office of Science and Technology, Health and Ecological Criteria Division, Washington, DC.
Giorgi, A. D. N., \& Tiraboschi, B. (1999). Evaluación experimental del efecto de dos grupos de macroinvertebrados (anfípodos y gasterópodos) sobre algas epífitas. Ecología Austral, 9(1), 35-44.

Halloy, S. R. P. (1991). Islands of Life at 6000 M Altitudethe Environment of the Highest Autotrophic Communities on Earth (Socompa Volcano, Andes). Arctic and Alpine Research, 23, 247-262.

Hijmans, R. J., Cameron, S. E., Parra, J. L., Jones, P. G., \& Jarvis, A. (2005). Very high resolution interpolated climate surfaces for global land areas. International Journal of Climatology, 25(15), 1965-1978.

Irons, J. G., Oswood, M. W., Stout, R. J., \& Pringle, C. M. (1994). Latitudinal patterns in leaf litter breakdown: is temperature really important? Freshwater Biology, $32(2), 401-411$.

Izquierdo, A. E., Foguet, J., \& Grau, H. R. (2015). Mapping and spatial characterization of Argentine High Andean peatbogs. Wetlands Ecology and Management, 1-14.

Jacobsen, D. (2003). Altitudinal changes in diversity of macroinvertebrates from small streams in the Ecuadorian Andes. Archiv für Hydrobiologie, 158(2), 145-167.

Jacobsen, D. (2008a). Tropical High-Altitude Streams. In D. Dudgeon (Ed.), Tropical stream ecology (pp. 219256). London, UK: Elsevier Science.

Jacobsen, D. (2008b). Low oxygen pressure as a driving factor for the altitudinal decline in taxon richness of stream macroinvertebrates. Oecologia, 154, 795-807.

Jacobsen, D., \& Marín, R. (2008). Bolivian Altiplano streams with low richness of macroinvertebrates and large diel fluctuations in temperature and dissolved oxygen. Aquatic Ecology, 42(4), 643-656.

Jacobsen, D., Rostgaard, S., \& Vasconez, J. J. (2003). Are macroinvertebrates in high altitude streams affected by oxygen deficiency? Freshwater Biology, 48(11), 2025-2032.

Jacobsen, D., Schultz, R., \& Encalada, A. (1997). Structure and diversity of stream macroinvertebrate assemblages: the effect of temperature with altitude and latitude. Freshwater Biology, 38, 247-261.

Janzen, D. H., Ataroff, M., Fariñas, M., Ricon, R. N., Soler, A., Soriano, P., \& Vera, M. (1976). Changes in the arthropod community along an elevational transect in the Venezuelan Andes. Biotropica, 8, 193-203.

Maldonado, M., \& Goitia, E. (2003). Las hidroecoregiones del Departamento de Cochabamba. Revista Boliviana de Ecología y Conservación Ambiental, 13, 117-141.

Maldonado, M., Maldonado-Ocampo, J. A., Ortega, H., Encalada, A. C., Carvajal-Vallejos, F. M., 
Rivadeneira, J. F., Acosta, F., Jacobsen, D., Crespo, A., \& Rivera-Rondón, C. A. (2011). Biodiversity in Aquatic Systems of the Tropical Andes. In S. K. Herzog, R. Martínez, P. M. Jorgensen, \& H. Tiessen (Eds). Climate Change and Biodiversity in the Tropical Andes Inter-American Institute for Global Change Research (IAI) and Scientific Committee on Problems of the Environment (SCOPE) (pp. 276294). São José dos Campos, Paris.

Maldonado-Ocampo, J., Ortega-Lara, A., Usma, J. S., Galvis, G., Villa-Navarro, F. A., Vásquez, L., PradaPedreros, S., \& Ardila, C. (2005). Peces de los Andes de Colombia. Bogotá: Instituto de Investigación de Recursos Biológicos Alexander von Humboldt.

Mani, M. S (1968). Ecology and biogeography of high altitude insects. The Hague: Dr. W. Junk.

McCoy, E. A. (1990). The distribution of insects along elevational gradients. Oikos, 58, 313-322.

McCune, B., \& Grace, J. B. (2002). Analysis of Ecological Communities. MJM Software Design, Gleneden Beach, OR.

Molina, C. I., Gibon, F. M., Pinto, J., \& Rosales, C. (2008). Estructura de macroinvertebrados acuáticos en un río altoandino de la Cordillera Real, Bolivia: Variación anual y longitudinal en relación a factores ambientales. Ecología Aplicada, 7 (1-2), 105-116.

Monaghan, K. A., Peck, M. R., Brewin, P. A., Masiero, M., Zarate, E., Turcotte, P., \& Ormerod, S. J. (2000). Macroinvertebrate distribution in Ecuadorian hill streams: the effects of altitude and land use. Archiv fur Hydrobiologie, 149, 421-440.

Moya, C., Valdovinos, C., Moraga, A., Romero, F., Debels, P., \& Oyanedel, A. (2009). Patrones de distribución espacial de ensambles de macroinvertebrados bentónicos de un sistema fluvial Andino Patagónico. Revista Chilena de Historia Natural, 82, 425-442.

Muñoz-Quesada, F. (2004). El Orden Trichoptera (Insecta) en Colombia, II: inmaduros y adultos, consideraciones generales. Insectos de Colombia, 3, 319-334.

Ortega, H., \& Hidalgo, M. (2008). Freshwater fishes and aquatic habitats in Perú: current knowledge and conservation. Aquatic Ecosystem Health and Management, 11, 257-271.

Pauli, H., Gottfried, M., Hohenwallner, D., Reiter, K., Casale, R., \& Grabherr, G. (2004). The GLORIA field manual. Multi-summit approach. European Commission. Luxembourg: Office for Official Publications of the European Communities.

Paoli, H. P., Elena, H. J., Mosciaro, M. J., Ledesma, F., \& Noé, Y. E. (2014). Caracterización de las cuencas hídricas de las provincias de Salta y Jujuy.
Rahbek, C. (1995). The elevational gradient of species richness: a uniform pattern? Ecography, 18, 200-205.

Rahbek, C. (2005). The role of spatial scale and the perception of largescale species-richness patterns. Ecology Letter, 8, 224-239.

Ramsay, P. M., \& Oxley, E. R. B. (1997). The growth composition of plant communities in the Ecuadorian paramos. Plant Ecology, 131, 173-192.

Reboratti, C. (2006). La situación ambiental en las ecoregiones Puna y Altos Andes. In A. Brown, U. Martínez Ortiz, M. Acerbi, J. Corcuera (Eds.), La Situación Ambiental Argentina 2005. Argentina: Fundación Vida Silvestre.

Reynaga, M. C., \& Dos Santos, D. A. (2012). Rasgos biológicos de macroinvertebrados de ríos subtropicales: patrones de variación a lo largo de gradientes ambientales espacio-temporales. Ecología Austral, $22,112-120$.

Rumrich, U., Lange-Bertalot, H., \& Rumrich, M. (2000). Diatoms of the Andes from Venezuela to Patagonia/ Tierra del Fuego. Iconographia Diatomologica, 9, 1-649.

Ruthsatz, B. (1978). Las plantas en cojín de los semidesiertos andinos del NOA. Darwiniana, 21(2-4), 491-540.

Ruthsatz, B. (2012). Vegetación y ecología de los bofedales altoandinos de Bolivia. Phytocoenologia, 42(3-4), 133-179.

Saigo, M., Marchese, M., \& Montalvo, L. (2009). Hábitos Alimentarios de Hyalella curvispina Shoema Ker, 1942 (Amphipoda : Gammaridea ) en ambientes leníticos de la llanura aluvial del río Paraná Medio. Natura Neotropicalis, 40(1-2), 43-59.

Sarmiento, J., \& Barrera, S. (1997). Observaciones preliminares sobre la ictiofauna de la vertiente oriental de los Andes. Revista Boliviana de Ecología y Conservación Ambiental, 2, 77-99.

Scheibler, E. E., Pozo, V., \& Paggi, A. C. (2008). Distribución espacio-temporal de larvas de Chironomidae (Diptera) en un arroyo andino (Uspallata, Mendoza, Argentina). Revista de la Sociedad Entomológica Argentina, 67(3-4), 45-58.

Segnini, S., \& Chacón, M. M. (2005). Caracterización Fisicoquímica del hábitat interno y ribereño de ríos Andinos en la cordillera de Mérida, Venezuela. Ecotropicos, 18(1), 38-61.

Seijmonsbegen, A. C., Sevink, J., Cammeratt, J., \& Recharte, J. (2010). A potential geoconservation map of the Lagunas Area, northern Peru. Environmental Conservation $37,107-115$. 
Seimon, T. A., Seimon, A., Daszak, P., Halloy, S. R. P., Schloegel, L. M., Aguilar, C. A., Sowell, P., Hyatt, A. D., Konecky, B., \& Simmons, J. E. (2007). Upward range extension of Andean anurans and chytridiomycosis to extreme elevations in response to tropical deglaciation. Global Change Biology, 12, 1-12.

Tejerina, E. G., \& Malizia, A. (2012). Chironomidae (Diptera) larvae assemblages differ along an altitudinal gradient and temporal periods in a subtropical montane stream in Northwest Argentina. Hydrobiologia, $686,41-54$.

Tripole, S., Vallania, E. A., \& Corigliano, M. C. (2008). Benthic macroinvertebrate tolerance to water acidity in the Grande river sub-basin (San Luis, Argentina). Limnetica, 27, 29-38.

Vannote, R. L., Minshall, G. W., Cummins, K. W., Sedell, J. R., \& Cushing, C. E. (1980). The River Continuum Concept. Canadian Journal of Fisheries and Aquatic Sciences, 37(1), 130-137.

Vinson, M. R., \& Hawkins, C. P. (1998). Biodiversity of stream insects: variation at local, basin, and regional scales. Annual Review of Entomology, 43, 271-293.

Walling, D. E., \& Webb, B. W. (1992). Water quality: physical characteristics. In P. Calow \& G. E. Petts
(Eds.). The Rivers Handbook (pp.48-72). Oxford, UK: Blackwell Scientific.

Wantzen, K. M., \& Wagner, R. (2006). Detritus processing by invertebrate shredders: a neotropical temperate comparison. Journal of the North American Benthological Society, 25(1), 216-232.

Ward, J. V. (1985). Thermal characteristics of running waters. Hydrobiologia, 125, 31-46.

Wasson, J. G., Guyot, J. L., Dejoux, C., \& Roche, M. A. (1989). Régimen térmico de los ríos de Bolivia.

Wesner, J. S. (2012). Emerging aquatic insects as predators in terrestrial systems across a gradient of stream temperature in North and South America. Freshwater Biology, 57(12), 2465-2474.

White, M. M., Chejlava, M., Fried, B., \& Sherma, J. (2007). The concentration of calcium carbonate in shells of freshwater snails. The American Malacological Bulletin, 22(1), 139-142.

Zúñiga, M. del C., Molineri, C., \& Domínguez, E. (2004). El orden Ephemeroptera (Insecta) en Colombia. In M. G. F. Fernández, G. Andrade y G. Amat García (Eds.), Insectos de Colombia (pp. 17-42). Bogotá: Academia Colombiana de Ciencias Exactas, Físicas y Naturales. 